\title{
A REMARK ON MULTIPLIER METHODS FOR NONLINEAR PROGRAMMING
}

\author{
M. Cirinà \\ Istituto Matematico del Politecnico \\ 10129 Torino, Italy
}

\begin{abstract}
This paper is concerned with certain aspects of multiplier methods where the solution of a constrained minimization problem is obtained by means of a sequence of unconstrained minimizations of an augmented Lagrangian $\mathrm{L}(\mathrm{x}, \mathrm{y}, \mathrm{r})$, followed each by an iteration on the La grange multipliex vector $y$. In spite of the growing recognition that multipliex methods are among the most effective constrained minimization methods, the value to be given to the penalty parameter $r$ does not seem yet to have received enough attention. This paper - related to work done recently by Bertsekas and Polyak - contains a result in such direction, namely the following one: if $G$ and $Q$ are given matrices and $Q$ is positive definite on the kernel of $G$, then $i t$ is produced $r *$ such that for all $r>r^{*}, Q+r G^{T}$ is positive definite on the whole space. Also we prove a lemma - related toa known one - about Hilbert space operators with uniformly bounded inverses, that may be useful in extending the result above to more general situations. To test the computational value of the estimate $r *$ arrived at here, a computer program is being tested and some numerical results are reported.
\end{abstract}

\section{INTRODUCTION}

Let $f, g_{1}, \ldots, g_{m}$ be real valued functions defined on $R^{n}, n \geqslant m>0$, and $g=\left(g_{1}, \ldots, g_{m}\right)$. Consider the problem

$$
\text { minimize } f(x) \text {, subject to } g(x)=0
$$

and the sequence of unconstrained minimizations

$$
L\left(x_{k}, y_{k}, r_{k}\right)=\min \left\{L\left(\cdot, y_{k}, r_{k}\right) \mid x \in R^{n}\right\}
$$

where $\left\{\mathrm{y}_{\mathrm{k}}\right\},\left\{\mathrm{r}_{\mathrm{k}}\right\}$ are to be chosen appropriately and the augmented Lagran gian L-cf. Arrow and Solow [1], Fletcher [5], Hestenes [6] and the work of Rockafellar, see $[10,11]$ and their references - is defined by 
(1.2) $\left.L(x, y, r)=f(x)+(y, g(x))+\frac{r}{2}\|g\|^{2},(x, y, r) \in R^{n} \times R^{m} \times\right] \circ, \infty[$.

The penalty method for solving (P) - see for instance Fiacco and McCor mick [4] - can be thought of as the minimizations (1.1) with the choice $y_{k}=0$ and $x_{k} \uparrow \infty$. The numerical difficulties (instability) that may arise in such procedure for " $r_{k}$ large" can in general be avoided or reduced by using the iteration

$$
y_{k+1}=y_{k}+r_{k} y\left(x_{k}\right)
$$

for which the condition $r_{k} A \infty$ is not necessary: this choice of $y_{k}$ characterizes the multiplier methods - see Hestenes [6] and Powell [9] - that are receiving growing recognition as one of the most effec tive class of methods for constrained minimization.

In the actual process of solving a specific problem (P) with such a scheme one has of course to decide which values the penalty parameter $r_{k}$ is to be given; it may be easily found out that haphazard guesses may lead to a substantial waste of time and effort: indeed, roughly speaking, when the violation of the constraints is penalized too little (1.1) may end up generating points away from the feasible set, and when it is penalized too much the points generated by (1.1) may have tenden cy to jump around.

In a recent paper, Bertsekas [ 3 ] has succeded in proving convergence and rate of convergence results for the multiplier method; actually, under the standard $2^{\text {nd }}$ order sufficiency conditions for $\bar{x}$ to be an iso lated local minimum (i.e. when - see Luenberger $[7]$, p.226 $f$ and $g$ are $C^{2}$ in a ball $B(\bar{x}, \varepsilon)$, at $\bar{x}$ the differential $d g$ of $g$ has rank $m$ and $d f+\vec{y}^{T} d g=0$ for some $\bar{y} \in R^{m}$, at $(\bar{x}, \bar{y}$, o) the hessian of $\mathrm{L}(\cdot, \overline{\mathrm{y}}, 0)$ is positive definite on the kernel of $\mathrm{dg}(\overline{\mathrm{x}})$ ) and under a Lipschitz condition on $d^{2} f, d^{2} g_{1}, \ldots, d^{2} g_{m}$ in $B(\bar{x}, \varepsilon)$ he proves that given a bounded subset $y \subseteq R^{\mathrm{m}} \exists r_{1} \geqslant 0$ and $M_{1} \geqslant 0$ such that for all $r \geqslant r_{I}$ and $y \in y$

(i) $\exists$ one minimizer $\mathrm{x}(\mathrm{y}, \mathrm{r})$ of $\mathrm{L}(\cdot, \mathrm{y}, \mathrm{r})$ in $\mathrm{B}(\overline{\mathrm{x}}, \varepsilon), \varepsilon>0$

$$
|x(y, r)-\bar{x}| \text { and }|y+r g(x(y, r))-\bar{y}| \leqslant \frac{M_{1}|y-\bar{y}|}{r} .
$$

In the proof, such conclusions are obtained by requiring - in particular - $r$ to be so large as to make

$$
d^{2} L(\bar{x}, \bar{y}, 0)+r d g^{T}(\bar{x}) d g(\bar{x}) \text { positive definite. }
$$


In section 2 it is given a computable estimate of $r *$ such that (1.4) holds for all $r>r *$; actually, we feel that such result (local estimar te theorem) is a special case of a global one.

The main difficulty for obtaining such more general result is that a cer tain set of matrices should possess uniformly bounded inverses. However, we shall not investigate the global problem here, except for setting it (in section 2), congecturing its solution and extending (in section, 3) a lemma in Polyak [8] about Hilbert space operators possessing uniformly bounded inverses, to a more general situation. Actualiy we feel that such more general lemma or some variant of it, besides having son me interest in themselves, may be useful for establishing our congectü re about the global result.

Finally some numerical results, obtained by making use of $r^{*}$ in a sim ple way within the standard multiplier method, are reported in section 4 and compared with analogous ones available in the letterature.

2. A LOCAL ESTIMATE OF $r *$

We shall first state the general problem we are concerned with. Let the norm \|\| be defined on the space of finite dimensional linear operators $L\left(R^{n}, R^{m}\right)$ by $\|A\|=\sup \left\{(A x, A x)^{1 / 2} /(x, x)^{1 / 2}: x \in R^{n}\right\}$, for $n \geqslant m>0$ con sider a map $G: X \subseteq R^{n} \rightarrow L\left(R^{n}, R^{m}\right)$ and let $N(G(x))$ be the kernel of $G(x)$, for each $x \in X$; also, consider a map $Q: Z=X \times y \subseteq R^{n} \times R^{m} \rightarrow L\left(R^{n}, R^{n}\right)$ and for $r$ real let $Q_{r}$ be defined by

$$
o_{r}(x, y)=Q(x, y)+r G^{T}(x) G(x),(x, y) \in X \times y
$$

The problem we are concerned with is the following:

if it is known that for $(x, y) \in Z, Q(x, y)$ has a coerciveness property on the subspace $N(G(x))$; when and how it possible to estimate $r$ such that for every $r>r * Q_{r}(x, y)$ has such coerciveness property on all of $\mathrm{R}^{\mathrm{n}}$ ?

Here we shall give an answer to such problem in the simpler case where the sets $x$ and $y$ contain just one point each.

Theorem 2.1 (Local estimate)

Let $n, m$ be positive integers $(n \geqslant m)$, and suppose that

(i) $G \in L\left(R^{n}, R^{m}\right)$ has rank $m$;

(ii) $Q \in I\left(R^{n}, R^{n}\right)$ is symmetric and $(Q s, s)>0$, all $s \in N(G), s \neq 0$. 
For $r$ real consider $Q_{r}=Q+r G^{T}$ and let $r *$ be defined by (2.3) be low. Then

$$
x>x^{*} \Rightarrow\left(Q_{r} s, s\right)>0 \text {,all } s \in \mathbb{R}^{n}, s \neq 0
$$

Remark 2.2. Since $Q_{r}$ is a matrix of numbers, to say that it is coercive it is the same as saying that it is positive definite.

Proof of theorem 2.1.

Let us observe that there are congruent transformations $B(\cdot) B^{T}$ that re duce $G^{T} G$ to a diagonal matrix; such transformations - by a classical re sult of Sylvester - leave invariant the number of positive and the number of zero eigenvalues. A natural choice for one such transformation is given by $B \in L\left(R^{n}, R^{n}\right)$ defined as

$$
B=\left[G^{T} \mid e_{j}, \ldots, e_{j}\right]_{n-m}^{-1}
$$

where $e_{j_{p}}$ is the $j_{p}$-th column of the identity matrix and $j_{p}$ is the row index of the p-th row of $\mathrm{G}^{\mathrm{T}}$ that is linearly dependent on the previous ones. Indeed it can be easily seen that such $B$ acts as left inverse of $\mathrm{G}$ and

$$
B G^{T} B^{T}=\left[\begin{array}{ll}
I & 0 \\
0 & 0
\end{array}\right], \text { I } \quad m \times m
$$

Let us now partition $\mathrm{BQ}_{\mathrm{r}} \mathrm{B}^{\mathrm{T}}$ symetrically

(2.2) $\quad \mathrm{BQ}_{r} \mathrm{~B}^{\mathrm{T}}=\mathrm{BQB} \mathrm{B}^{\mathrm{T}}+\left[\begin{array}{cc}r I & 0 \\ 0 & 0\end{array}\right]=\left[\begin{array}{cc}\mathrm{P}_{r} & \Pi^{\mathrm{T}} \\ \Pi & \mathrm{p}\end{array}\right], \mathrm{P}_{r} \quad \mathrm{~m} \times \mathrm{m}$;

put

$$
M(p ; \pi)= \begin{cases}2\left\|p^{-1}\right\|\|\pi\|^{2}-2\|\pi\|+\|p\| & \text { if } m<n \\ 0 & \text { else }\end{cases}
$$

and define $x^{*}$ by

$$
\text { (2.3) } x^{*}=\max \left\{0, \max _{1 \leqslant i \leqslant m}\left[-\left(\mathrm{BQB}^{\mathrm{T}}\right)_{i i}+\sum_{j=1, \neq i}^{\mathrm{m}}\left|\left(\mathrm{BQB}^{\mathrm{T}}\right)_{i j}\right|+M\right]\right\} .
$$

We claim that such $r *$ will do; indeed let $r>r *$ be given. It has to be shown that
(2.4)
$\left(s, Q_{r} s\right)>0$
all $s \in R^{n}, s \neq 0$. 
Since $G^{T} G$ is positive semidefinite, from the definition of $Q_{r}$ it is seen that (2.4) with $r \geq 0$ holds if $s$ is restricted to the kernel of $G$ i.e.

$$
\left(s, Q_{r} s\right)>0, \text { all } s \in N(G), \quad s \neq 0 \text {; }
$$

for $s \in R^{n}$ let $w \in R^{n}$ be defined by $s=B^{T} w$, call $w^{\prime}$ (respectively $w^{\prime \prime}$ ) the vector formed by the first $m$ (last $n-m$ ) components of $w$ and observe that

$$
\left(s, Q_{r} s\right)=\left(w, B_{r} B^{T} w\right)=\left(w^{\prime}, P_{r^{\prime}} w^{\prime}\right)+2\left(w^{\prime \prime}, \Pi w^{\prime}\right)+\left(w^{\prime \prime}, p w^{\prime \prime}\right)
$$

implies in view of the consequence of $(2.5)$

$$
\mathbf{s} \in \mathrm{N}(\mathrm{G}) \quad \Leftrightarrow \mathrm{w}^{\prime}=0,
$$

that the submatrix $\mathrm{p}$ is positive definite; thus $\mathrm{M}$ is a well defined real number.

We now claim that for $w \in R^{n}$ with $(w, w)=1=\left\|_{w^{\prime}}\right\|^{2}+\left\|_{w}\right\|^{2}$ one has

$$
\text { (2.6) }\left(w, B Q_{r} B^{T} w\right) \geqslant\left(\lambda\left(P_{r}\right)-\|p\|+2\|\Pi\|\right)\left\|w^{\prime}\right\|^{2}-2 \sqrt{2}\|\Pi\|\left\|w^{\prime}\right\|^{2}+\| p^{-l_{\|}}-1
$$

where $\lambda(\cdot)$ denotes the least eigenvalue of the argument; indeed using the Cauchy-Schwarz inequality, the fact that $(1-\sqrt{2} t)^{2} \geqslant 0$ for $t$ real and that $\left(w^{\prime}, p w^{\prime \prime}\right) \geqslant \lambda(p)\left(1-\left\|_{w} \cdot\right\|^{2}\right)$ one obtains

$$
\left(w, B Q_{r} B^{T} w\right) \geqslant\left(\lambda\left(P_{r}\right)-\lambda(p)+2\|\Pi\|\right)\left\|W^{\prime}\right\|^{2}-2 \sqrt{2}\|\pi\|\left\|_{W^{\prime}}\right\|+\lambda(p)
$$

and hence $(2.6)$, because $\left\|\underline{p}^{-1}\right\|^{-1}=\lambda(p) \leqslant\|\underline{p}\|$.

From (2.6) one has

(2.7) $2\|\pi\|^{2}-\left(\lambda\left(P_{r}\right)-\|p\|+2\|\pi\|\right)\left\|p^{-1}\right\|^{-1}<0 \Rightarrow\left(w, B Q_{r} B^{T} w\right)>0$, all $w,\|w\|=1$

from which it is seen that the proof of the theorem is completed as soon as it is shown that

$$
\lambda\left(\mathrm{P}_{r}\right)>\mathrm{M}(\mathrm{p} ; \mathrm{I}) \text {; }
$$

this inequality does indeed hold because it follows from Gershgorin's theorem and the choice of $r *$ : in fact, there is an integer $i(1 \leqslant i \leqslant m)$ such that

$$
-\lambda\left(P_{Y}\right)+\left[\left(B Q B^{T}\right)_{i i}+r\right] \leqslant \sum_{j=1, f i}^{m}\left|\left(B O B^{T}\right)_{i j}\right|,
$$

and this inequality implies (2.8) because $r>r^{*}$. 
If the condition " $X$ and $Y$ contain just one point each" is dropped and the hypotheses of theorem 2.1 are somewhat strengthened so as to make the matrices $B(x)$ (i.e. the left inverses of $G^{T}(x), x \in X$ ) uniformly bounded in $x$, we concecture that a result similar to the one above should hold. In the following section we shall examine some conditions insuring that linear operators depending on parameters possess uniformly bounded inverses .

\section{UNIFORMLY BOUNDED INVERSES}

In this section we generalize a result of Polyak, [ 8 ] lemma 1, about Hilbert space operators with uniform 1 bounded inverses and then give a variant of it.

Let $H$, $h$ be real Hilbert spaces; their inner product as well as the intm duced norms will be denoted by the same symbols $(\cdot, \cdot)$ and $\|\cdot\|$, when there is no risk of misunderstanding; let the product space $\mathrm{H} \times \mathrm{h}$ be normed by $\|\cdot\|^{2}=\|\cdot\|_{H}^{2}+\|\cdot\|_{h}^{2}$, the space $\mathrm{L}(\mathrm{H}, \mathrm{h})$ of bounded linear open rators from $\mathrm{H}$ to $\mathrm{h}$ be normed by $\|\mathrm{C}\|=\sup \left\{\|\mathrm{Cs}\|_{\mathrm{h}} /\left\|_{\mathrm{s}}\right\|_{\mathrm{H}}: \mathrm{s} \neq 0\right\}$, and let $x, Z, R$ be subsets of the Euclidean spaces $R^{\nu}, R^{\mu}$ and $R^{P}$ respecti vely. Suppose

(3.1) $\left\{C_{X}: x \in X\right\}$ is a bounded family of operators $C_{X} \in I(H, h)$ satisfying $\left\|C_{x}^{*} s\right\| \geqslant \sqrt{k},\|s\|$, all $s \in h$ and $x \in X$, some $k_{1}>0$, where $c_{x}^{*}$ denotes the adjoint of $\mathrm{C}_{\mathrm{X}}$;

(3.2) $\left\{U_{z}: z \in Z\right\}$ is a bounded family of symmetric operators $U_{z} \in \mathrm{L}(\mathrm{H}, \mathrm{H})$ satisfying $\left(\mathrm{U}_{\mathrm{z}} \mathrm{s}, \mathrm{s}\right) \geqslant \mathrm{k}_{2}\|\mathrm{~s}\|^{2}$, all $\mathrm{s} \in \mathrm{H}, \mathrm{z} \in \mathrm{Z}$, some $\mathrm{k}_{2}>0$; let $k_{3}$ be a positive number such that $\left\|u_{z}\right\| \leqslant k_{3}$, all $z \in z$;

(3.3) $\left\{u_{r}: r \in R\right\}$ is a family of operators $u_{r} \in I(h, h)$ satisfying $\left(u_{r} s, s\right) \leqslant k_{4}\left\|_{s}\right\|^{2}$, all $s \in h$ and $r \in R$, with $k_{4}$ real.

For each $(x, z, r) \in P=X \times Z \times R$, consider the operator $A=A, x, z, r: H \times h \rightarrow H \times h$ defined by

$$
A=\left[\begin{array}{ll}
u_{z} & C_{x} \\
c_{x} & u_{r}
\end{array}\right]
$$

If the families (3.1), (3.2) reduce to a single operator and if the $u_{x}{ }^{\prime} s$ are the specific negative operators $-I / x$ where $I$ is the identity and $r \in 10, \infty[$, Polyak proves in [8] that the inverse of $A$ exists and is 
bounded uniformly in $r$. Here we obtain a similar result for the more ge neral situation (3.1), (3.2) and (3.3), where in particular (3.3) does not esclude $u_{r}$ to be the null operator, a case important for some nonli near programming methods, and indeed allows the operators $u_{r}$ to be some what positive; the simplicity of the proof is enhanced by the explicit exhibition of the inverse of $A$.

Lemma 3.1 (Formula for the inverse operator)

Suppose that (3.1), (3.2), and (3.3) with $k_{4}<k_{1} / k_{3}$ hold; for each $(x, z, r) \in P$ define the operator $F=F_{x, z_{p} r}$ by

$$
F_{X, z, r}=\left[\begin{array}{cc}
U^{-1}+S^{-1} S^{*} & -S v^{-1} \\
-v^{-1} S^{*} & v^{-1}
\end{array}\right]
$$

where $U^{-1}=U_{z}^{-1}$ and the operators $S=S_{x, z}, v=v_{x, z, r}$ are defined by $s=U_{z}^{-1} C_{x}^{*}, v=u_{x}-C_{x} U_{z}^{-1} C_{x}^{*}$. Then

(i) $\exists$ k real: $\left\|F_{x, z, r}\right\| \leqslant K \forall(x, z, r) \in P$;

(ii) $\forall(x, z, r) \in P: F_{x, z, r}$ is the inverse of $A_{x, z, r}$.

Proof

In view of $(3.1),(3.3)$ and the choice of $k_{4}$, the family of operators on $h\left\{-v_{x, z}, r\right\}$ is uniformly coercive because

$$
\begin{aligned}
\left(-\mathrm{v}_{\mathrm{x}, \mathrm{z}, \mathrm{r}} \mathrm{s}, \mathrm{s}\right) & =-\left(\mathrm{u}_{\mathrm{r}} \mathrm{s}, \mathrm{s}\right)+\left(\mathrm{C}_{\mathrm{x}} \mathrm{U}_{\mathrm{z}}^{-1} \mathrm{C}_{\mathrm{x}}^{*} \mathrm{~s}, \mathrm{~s}\right) \geqslant-\mathrm{k}_{4}\|\mathrm{~s}\|^{2}+\frac{1}{\left\|\mathrm{U}_{\mathrm{z}}\right\| \mathrm{C}_{\mathrm{x}}^{*} \|^{2}} \\
& \geqslant\left(\frac{\mathrm{k}_{1}}{\mathrm{k}_{3}}-\mathrm{k}_{4}\right)\|\mathrm{s}\|^{2}, \text { all } \mathrm{s} \in \mathrm{h} \text { and }(\mathrm{x}, \mathrm{z}, \mathrm{r}) \in \mathrm{p}
\end{aligned}
$$

and hence $\left\{v_{x, z, r}^{-1}\right\}$ is a bounded family because (3.5) implies, in addition to the existence of the inverses, that

$$
\left\|\mathrm{v}_{\mathrm{x}, \mathrm{z}, \mathrm{r}}^{-1}\right\| \leqslant \frac{1}{\left(\mathrm{k}_{1} / \mathrm{k}_{3}\right)-\mathrm{k}_{4}}, \quad \text { all }(\mathrm{x}, \mathrm{z}, \mathrm{r}) \in P
$$

thus the operator $F_{x, z, r}$ is well defined. A direct check of its product with $A x, z, r$ shows that indeed (ii)holds. Also $\left\{U_{z}^{-1}\right\}$ is a bounded subset of $L(H, H)$ because the uniform coerciveness condition in (3.2) implies

$$
\left\|U_{z}^{-1}\right\| \leqslant \frac{1}{k_{2}} \quad, \text { all } z \in Z
$$

in view of (3.6), (3.8) and (3.1), all the operators appearing in the definition (3.4) belong to bounded families, hence the uniform boundedness conclusion (i) is a simple consequence of the fact that

$$
\left\|F_{x, z, r}\right\| \leqslant\left(\left\|U^{-1}+S v^{-1} S *\right\|+\left\|S v^{-1}\right\|+\left\|v^{-1} S *\right\|+\left\|v^{-1}\right\|\right) \|_{x, z, r} \text {. }
$$


The lemma is thus established.

We shall now give a variant of lemma 1 : roughly speaking, if the set $\left\{u_{r}\right\}$ is sufficienty coercive, the hypotheses on $\left[c_{x}\right\}$ can be weakened. More precisely, let us assume that

(3.I) $\left\{C_{x}: x \in X\right\}$ is a bounded subset of $L(H, h)$; let $k_{5}$ be a number such that $\left\|C_{x}^{*}\right\| \leqslant k_{5}$, all $x \in X$;

$(3.3)^{\prime}\left\{u_{r}: r \in R\right\}$ is a subset of $L(h, h)$ satisfying: $\left(u_{r} s, s\right) \geqslant k_{6}\|s\|^{2}$, all $\mathrm{s} \in \mathrm{h}$ and $\mathrm{r} \in R, \mathrm{k}_{6}>0$.

Lemma 3.2 .

Under the hypotheses $(3.1)^{*},(3.2)$, and (3.3)' with $k_{6}>k_{5}^{2} / k_{2}$ the conclusions of lemma 3.1 hold.

Proof

Observe that

$$
\begin{aligned}
\left(v_{x, z, r} s, s\right) & =\left(u_{x} s, s\right)-\left(C_{x} U_{z}^{-1} C_{x}^{*} s, s\right) \geqslant k_{6}\|s\|^{2}-\left\|U_{z}^{-1}\right\|\left\|C_{x}^{*}\right\|^{2}\|s\|^{2} \\
& \geqslant\left(k_{6}-k_{5}^{2} / k_{2}\right)\|s\|^{2}
\end{aligned}
$$

holds for all $s \in h$ and $(x, z, r) \in P$; hence

$$
\left\|v_{x, z, r}^{-1}\right\| \leqslant\left(k_{6}-k_{5}^{2} / k_{2}\right)^{-1}
$$

From now on the proof continues as in lemma 3.1 and is omitted.

Let us now return to the general problem formulated at the beginning of section 2 and make more precise our congecture about its solution. In view of the lemmas above, the rank condition as well as the positive definiteness condition of theorem 2.1 should be strengthened so as to hold uniformly in the parameters. More precisely we congecture that under the following type of hypotheses

- the map $G$ is bounded and satisfies: $\left\|G^{T}(x) s\right\| \geqslant k_{1}\|s\|$, for all $s \in R^{m}$, all $x \in X$, and some $k_{1}>0$, where $G^{T}(x)$ denotes the transpose of $\mathrm{G}(\mathrm{x})$;

- the map $Q$ is bounded, its values are symmetric (matrices), and satisfies $(Q(x ; y) s, s) \geqslant k_{2}\|s\|^{2}$, for all $s \in N(G(x))$, all $(x ; y) \in X \times y$, and some $\mathrm{k}_{2}>0$;

it is likely to hold a global estimate of the following type

$$
r^{*}=\max \left\{0, \sup _{x, y} \max _{1 \leqslant i \leqslant m}\left[-\left(B Q B^{T} \mid x, y\right)_{i i}+\sum_{j=1, \neq i}^{m}\left|\left(B Q B^{T} \mid x, y\right)_{i j}\right|+\hat{M}\right]\right\} .
$$


4. SOME NUMERICAL RESULTS

The estimate of the penalty parameter arrived at here has been used in a simple way - within the standard multiplier method scheme, and imple mented in a computer program. This latter has been tested in particular on the following example of constrained minimization problem (P):

(4.1) minimize $f(x)=x_{1} x_{2} x_{3} x_{4} x_{5}$, subject to

$$
\begin{aligned}
& g_{1}(x)=x_{1}^{2}+x_{2}^{2}+x_{3}^{2}+x_{4}^{2}+x_{5}^{2}-10=0 \\
& g_{2}(x)=x_{2} x_{3}-5 x_{4} x_{1}=0 \\
& g_{3}(x)=x_{5}^{3}+x_{2}^{3}+1=0
\end{aligned}
$$

whose solution (to 4 figures) is

$$
f(\bar{x})=-2.920, \bar{x}=(-0.7636,1.596,1.827,-0.7636,-1.717) .
$$

Numerical results for (4.1) were given originally by Powel1 [9] and subsequently by Asaadi [2] where, incidentally, there is a misprint in $g_{2}\left(x_{2}-x_{3}\right.$ instead of $\left.x_{2} x_{3}\right)$. Actually in [2], the initial point $x_{0}$ used in solving (4.1) is declared

$$
x_{0}=(-1,1.5,2,-1,-2),
$$

and (4.1) is among a number of examples solved by means of a few different nonlinear programs with the purpose of comparing their efficiency. Let $\mathrm{Ni}$ stand for number of iterations (outer iterations if there are as in the multiplier method inner iterations i.e. within each unconstrained minimization) and $\mathrm{Ne}$ stand for number of function evaluations. From [2] one sees that to solve $(4.1)$ to 4 figures ( $\mathrm{Ni} ; \mathrm{Ne}$ ) is

- $(7,148)$ with the method of Kowalik, Osborne and Ryan

- $(6,90)$ with Powell's version of the multiplier method

- $(6,126)$ with Fiacco and McCormick penalty method (SUMT).

By using the minimizations (1.2) with the iterations (1.3) initiated at $y_{0}=0$ and with the penalty parameter $r_{k}$ equal for all $k$ to the constant $r *$ computed in correspondance of the initial point, we have obtained the solution with $(\mathrm{Ni} ; \mathrm{Ne})=(3 ; 78)$. The usefulness of inserting the estimate $r *$, is not however in such possible slight imrrovement of the efficiency in terms of "number of function evaluations" as used in [2]; actually in [2] it is not said how has been fixed $r_{k}$ nor are tabulated initial guesses if any - i.e. minimizations (1.2) and iteration (1.3) that might have been necessary in order to find "good values" of the penalty parameter, for instance in the computations (4.2); such possible 
guess work, that need not indeed be negligible, is reduced or avoided by using an estimate such as $r *$.

\section{REFERENCES}

[1] Arrow K. J. and Solow, R. M., "Gradient Methods for Constrained Maxima with Weakened Assumptions", in Studies in Linear and Nonlinear Programming, Arrow, K., Hurwicz, L., and Uzawa, H. (eds.), Stanford University Press, Stanford, 1958.

[2] Asaadi J., "A Computational Comparison of some Non-linear Programs", Math. Prog., Vol. 4, 1973, pp. 144-154.

[3] Bertsekas D. P., "On Penalty and Multiplier Methods for Constrained Minimization", SIAM J. on Control, to appear.

[4] Fiacco A.V. and McCormick G. P., "Nonlinear Programming: Sequential Unconstrained Minimization Techniques", J. Wiley, New York, 1968.

[5] Fletcher R., "A class of methods for nonlinear programming with termination and convergence properties", in Integer and Nonlinear Programming, J. Abadie (editor), North-Holland, 1970.

[6] Hestenes M.R., "Multiplier and Gradient Methods", Journal of Optimi zation Theory and Applications, Vol. 4, No. 5, 1969; pp. 303-320.

[7] Luenberger D.G., "Introduction to Linear and Nonlinear Programing", Addison-Wesley, 1973 .

[8] Polyak B.T., "The Convergence Rate of the Penalty Function Method", Zh. Vychisl. Mat. Mat. Fiz., Vol. 11, No. 1, 1971, pp. 3-11.

[9] Powell M.J.D., "A Method for Nonlinear Constraints in Minimization Problems", in Optimization, R. Fletcher (ed.), Academic Press, pp. 283-298.

[10] Rockafellar R.T., "Augmented Lagrange Multiplier Functions and Duality in Nonconvex Programming", SIAM J. Control, Vol. 12, No.2, 1974 .

[11] Rockafellar R.T., "New applications of duality in convex programming", written version of talk at 7 th International Symposium on Math. Programming (the Hague, 1970) and elsewhere, published in the Proc. of the 4 th Conference on Probability (Brasov, Romania, $1 9 7 1 \longdiv { \text { . } }$ 